\title{
MAXIMUM ALLOWABLE DYNAMIC LOAD OF MOBILE MANIPULATORS WITH STABILITY CONSIDERATION
}

\author{
H. R. HEIDARY \\ College of Mechanical Engineering, \\ Malayer University, Malayer, Iran, \\ e-mail:hr_heidari@iust.ac.ir
}

[Received 06 March 2015. Accepted 15 June 2015]

\begin{abstract}
High payload to mass ratio is one of the advantages of mobile robot manipulators. In this paper, a general formula for finding the maximum allowable dynamic load (MADL) of wheeled mobile robot is presented. Mobile manipulators operating in field environments will be required to manipulate large loads, and to perform such tasks on uneven terrain, which may cause the system to reach dangerous tip-over instability. Therefore, the method is expanded for finding the MADL of mobile manipulators with stability consideration. Moment-Height Stability (MHS) criterion is used as an index for the system stability. Full dynamic model of wheeled mobile base and mounted manipulator is considered with respect to the dynamic of non-holonomic constraint. Then, a method for determination of the maximum allowable loads is described, subject to actuator constraints and by imposing the stability limitation as a new constraint. The actuator torque constraint is applied by using a speed-torque characteristics curve of a typical DC motor. In order to verify the effectiveness of the presented algorithm, several simulation studies considering a two-link planar manipulator, mounted on a mobile base are presented and the results are discussed.

KEY WORDS: Non-holonomic mobile manipulator, maximum dynamic load, stability constraint.
\end{abstract}

\section{Introduction}

High payload to mass ratio is one of the advantages of mobile robot manipulators. In traditional mobile manipulators, the maximum allowable dynamic load is usually defined as the maximum load, which a robot can repeatedly lift and carry on the fully extended configuration, while the dynamics of both the load and the mobile manipulator itself must be taken into account. 
The maximum load-carrying capacity, which can be achieved by a robotic manipulator during a given trajectory is limited by a number of factors. Probably, the most important factors are the actuator limitations and the stability constraint.

The research interest in mobile manipulator, i. e., light and large dimension robotic, has increased significantly during the last few years. Mobile manipulators have important application in space exploration, manufacturing automation, construction, undersea, nuclear contaminated environments, and many other areas. Major advantages of mobile manipulator include, but not limited to, small mass, fast motion, and large force to mass ratio, which are reflected directly in the reduced energy consumption, increased productivity, and enhanced payload capacity. On the other hand, the effect of the manipulator and the payload movement, fast maneuver and moving on an uneven terrain, may cause the base to turn over, particularly for non-holonomic mobile manipulator, when operating high speed with long arm. Therefore, the stability of the mobile manipulator is the most important constraints for finding the MADL or enhancement of the payload capacity on a given trajectory. The mobile manipulators are usually "power on board" with limited torque capacity. Hence, using light and small platforms and motor actuators will help a mobile manipulator to work in an energy-efficient manner. On the other side, smaller actuators reduce the torque capacity of the actuators and limit the capability of carrying heavy loads. Consequently, a more successful approach should maximize the load carrying capacity of the mobile manipulator, subjected to the constraints imposed by torque capacity of joint actuators and stability of the system on a given trajectory.

Many approaches have been taken to the development of mobile manipulators $[2,13,15]$. Seraji [13] presented a simple on-line approach for motion control of mobile manipulators, using augmented Jacobian matrices. The approach used additional kinematics constraints to be met for the manipulator configuration, which can be equally applied to non-holonomic and holonomic mobile robots. Yamamoto and Yun [15] focused their research on the modelling and compensation of the dynamic interaction between the manipulator and the mobile platform of a mobile manipulator and developed a coordination algorithm, based on the concept of a preferred operating. Chen and Zalzala [2] have offered an approach for the modelling and motion planning of a mobile manipulator system with a non-holonomic constraint. The Newton-Euler equations are used to obtain the complete dynamics of the system.

Several researches have tried to establish the maximum load-carrying capacity of mobile manipulators. Wang and Ravani [14] studied and formu- 
lated the load carrying capacity of a manipulator on a given dynamic robot trajectory. The formulation associated with the dynamic load carrying capacity (DLCC) is considered with both joint actuator torque and joint variable constraints throughout the trajectory. Korayem and Ghariblu [7] developed an algorithm in order to find the DLCC of rigid mobile manipulators on a given trajectory. Korayem and Heidari [8] computed the maximum allowable dynamic load (MADL) of a moving base flexible manipulator. The accuracy and the torque constraints are taken into account for the proposed algorithm during motion for a given trajectory.

On the other point of view, some researchers have studied the stability of the mobile manipulators. For instance, Arakawa and Fukuda [1] presented a center of gravity control method to prevent a mobile manipulator from falling down, but the method discusses only the static stability. Ghasempoor and Sepehri [3] extended the energy stability method by Messuri and Klein [10] to quantitatively show the effects of top-heaviness and sloping ground. The extension can be used as an off-line tool to provide the designers with an inexpensive and fast method of maintaining the stability of mobile manipulators. Papadopoulos and Rey [12] have proposed a new tip over stability measure, the Force-Angle stability measure, which is easily computed and sensitive to topheaviness. The proposed measure has a simple geometric interpretation and applicable to dynamic systems, subjected to inertial loads and external forces. Li [9] has used the normal forces between the rear/front tires and ground for checking the static and dynamic stability of mobile manipulators.

The other approach is using zero moment point (ZMP), which, for the first time, Huang and Sugano [5] employed for tip over prevention of mobile manipulators. The valid stable region has been investigated for a mobile manipulator based on the ZMP criterion. But the inertia effect of rigid body has not been considered. Kim et al. [6] have proposed an online compensation algorithm to include the mass moment of inertia the original formulation of ZMP for the dynamic stability of the mobile manipulators. It should be mentioned, that in mobile manipulators, especially in manipulating heavy objects, the center-of-mass (C.M.) can be variable. But the ZMP criterion, in its basic format is insensitive to the system C.M. height. Moreover, the ZMP disregards the significant factor of the mass moment of inertia of the mobile base and does not provide any specific indication about severity of the system instability at all [4]. More recently, an efficient metric has been suggested by Moosavian and Alipour [11], which is called as moment-height stability (MHS) measure. This measure is based on stabilizing and destabilizing moments exerted on the moving base, which provides the system mobility. Also, the proposed metric is 
compared with other measures, such as Energy-Equilibrium Plan, Force-Angle, ZMP, and the efficiency of the MHS is superior over the others.

This paper presents a more accurate way of computing the "maximum load" of the non-holonomic mobile manipulator considering the dynamic stability. First, the equations of motion are derived taking into account full dynamic model of wheeled mobile base and mounted manipulator with respect to the dynamic of non-holonomic constraint. Then, a method for determination of the maximum allowable dynamic load for mobile robot is described with attention to actuator and overturning stability constraints on a given trajectory. MHS criterion is used as an index for the system stability. Finally, numerical examples considering a two-link mobile manipulator while travelling on a clothoid path and slope terrain are presented. The obtained simulation results demonstrate the accuracy and merits of the proposed method.

\section{Kinematics and dynamic model of the mobile manipulator}

In this section, a mobile manipulator comprising a manipulator arm mounted on a non-holonomic mobile base is considered, as shown in Fig. 1. The motion of the system has to be decomposed into the motion of the manipulator and the motion of the base. That is, the desired position of the manipulator's end effector can be defined as follows:

$$
x_{e}=x_{b}\left(q_{b}\right)+x_{m / b}\left(q_{m}\right)
$$

where $x_{e}$ and $x_{b}$ are the position of the end effector and the base in the inertial coordinate system. $x_{m / b}$ is the position vector of manipulator with respect to the base, also $q_{b}$ and $q_{m}$ are generalized variables of the base configuration space and the arms' space.

The considered system consists of two - DOF serial manipulator arms and a 5-DOF mobile base, which in reality the mobile base is with two degrees of freedom, because there are three restrictions of the non-holonom base. Manipulators' joints are revolute with a single degree of freedom. However, the developed methodology can be applied equally well to systems with n-link manipulators. The unidirectional platform, shown in Fig. 2, is a typical example of a non-holonomic WMR, which has two rear driving wheels and two castor wheels. The two driving wheels are powered by DC motors and possess the same wheel radius, $r$. Point $P_{b}$ is the origin of WMR axis, which is located at the intersection of the longitudinal $x$-axis and the lateral $y$-axis. $L_{0}$ and $b$ are length and width or WMR body, respectively. The origin of the inertial frame $\{X, Y\}$ is shown as $O$ and as such allows the pose of the WMR to be completely 


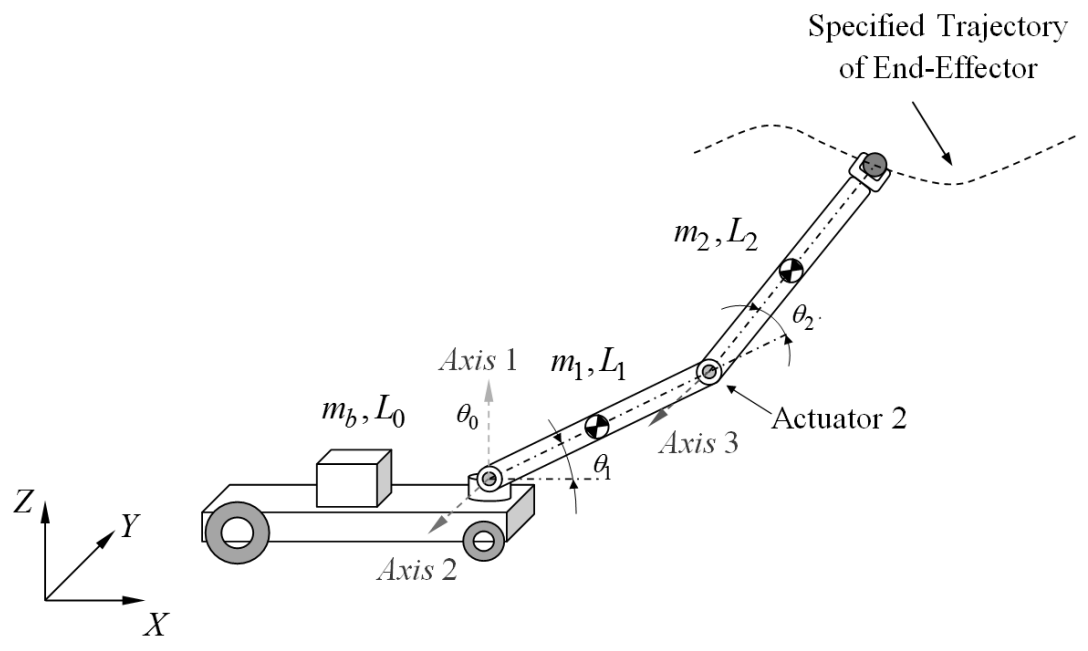

Fig. 1. Schematic view of a mobile manipulator

specified through the following vector of generalized coordinates with respect to $\{X, Y\}, q_{b}=\left[\begin{array}{lllll}X_{b} & Y_{b} & \varphi & \theta_{r} & \theta_{l}\end{array}\right]$, where $X_{b}$ and $Y_{b}$ are the coordinates of the center of mass. The orientation of the WMR frame from the inertial frame is denoted as $\varphi . \theta_{r}$ and $\theta_{l}$ are the angular displacements of right and left driving wheel, respectively. Due to the non-holonomic nature of the system, the constraint equation obeys the ideal no-slip condition. The rolling and the knife edge constraint equations for this system are given by Yamamoto and Yun [15]. In the next paragraph, these constraints will be explained.

The Lagrangian method is utilized to formulate the dynamic equations, governing the motion of the mobile manipulator systems. In order to derive dynamic equations, the kinetic energy and the potential energy are computed for the entire system. After calculation of these energies, by applying the Lagrangian multipliers procedure and performing some algebraic manipulations, the compact form of the governing equations of two-link mobile manipulator can be obtained from:

$$
\begin{array}{r}
{\left[\begin{array}{cc}
M_{b b} & M_{b m} \\
M_{m b} & M_{m m}
\end{array}\right]\left[\begin{array}{c}
\ddot{q}_{b} \\
\ddot{q}_{m}
\end{array}\right]+\left[\begin{array}{c}
C_{b}\left(q_{b}, q_{m}, \dot{q}_{b}, \dot{q}_{m}\right) \\
C_{m}\left(q_{b}, q_{m}, \dot{q}_{b}, \dot{q}_{m}\right)
\end{array}\right]} \\
+\left[\begin{array}{c}
G_{b}\left(q_{b}, q_{m}\right) \\
G_{m}\left(q_{b}, q_{m}\right)
\end{array}\right]=\left[\begin{array}{c}
F_{b}-\Lambda^{T}(q) \lambda \\
F_{m}
\end{array}\right],
\end{array}
$$

where $[M]$ is the mass matrix, $\{C\}$ is the vector of Coriolis and centrifugal forces, $\{G\}$ describes the gravity effects, and $[\Lambda]$ denotes to non-holonomic 


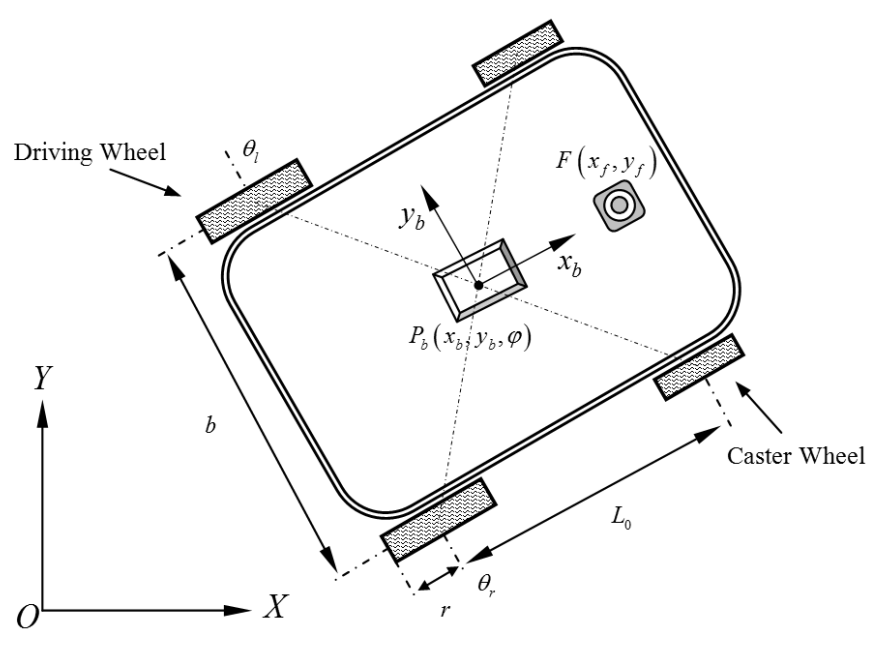

Fig. 2. Nonholonomic Wheeled Mobile Robot Platform

constraints. The generalized coordinates, $\{q\}$, and the generalized force, $\{F\}$, are the following vectors:

$$
\begin{aligned}
& \{q\}=\left[\begin{array}{lll}
q_{b} & , & q_{m}
\end{array}\right]^{T}=\left[\begin{array}{lllllllll}
X_{f} & Y_{f} & \varphi & \theta_{r} & \theta_{l} & , & \theta_{0} & \theta_{1} & \theta_{2}
\end{array}\right]^{T} \\
& \{F\}=\left[\begin{array}{lll}
F_{b} & , & F_{m}
\end{array}\right]^{T}=\left[\begin{array}{lllllllll}
0 & 0 & 0 & \tau_{w r} & \tau_{w l} & , & \tau_{0} & \tau_{1} & \tau_{2}
\end{array}\right]^{T}
\end{aligned}
$$

The generalized coordinates $q_{b}, q_{m}$ are defining the mobile base motion, rigid body motion of manipulator, respectively.

The non-holonomy in mobile robotics causes some difficulties in modelling and motion planning, but it reduces the number of driving motors, needed for the motion of the platform and a non-holonomic mobile platform is more cost efficient. There are three constraints in motion of the non-holonomic mobile platform: the first one is that the platform must move in the direction of the axis of symmetry and the other two constraints are the rolling constraints not allowing them to slip. The non-holonomic constraints to which the platform is subjected are given as

$$
\begin{aligned}
& \dot{Y}_{f} \cos \varphi-\dot{X}_{f} \sin \varphi-d \dot{\varphi}=0 \\
& \dot{Y}_{f} \sin \varphi+\dot{X}_{f} \cos \varphi+b \dot{\varphi}=r \dot{\theta}_{r} \\
& \dot{Y}_{f} \sin \varphi+\dot{X}_{f} \cos \varphi-b \dot{\varphi}=r \dot{\theta}_{l}
\end{aligned}
$$

The three constraints can be written in the compact form:

$$
\Lambda(q) \dot{q}=0
$$


And:

$$
\Lambda=\left[\begin{array}{cccccccc}
-\sin \varphi & \cos \varphi & -d & 0 & 0 & 0 & 0 & 0 \\
-\cos \varphi & -\sin \varphi & -b & r & 0 & 0 & 0 & 0 \\
-\cos \varphi & -\sin \varphi & b & 0 & r & 0 & 0 & 0
\end{array}\right]
$$

where $r$ is the radius of the driving wheel, $b$ is the distance of two wheels and $\mathrm{d}$ is the distance between front and rear wheels. By defining the $[B(q)]$ matrix, which is the null space of $[\Lambda(q)]$ matrix, the Lagrange multipliers can be eliminated:

$$
\Lambda(q) B(q)=0 .
$$

One choice of $B(q)$ is as follows:

(9)

$$
B=\left[\begin{array}{ccccc}
(r / 2 b)(b \cos \varphi-d \sin \varphi) & (r / 2 b)(b \cos \varphi+d \sin \varphi) & 0 & 0 & 0 \\
(r / 2 b)(b \sin \varphi+d \cos \varphi) & (r / 2 b)(b \sin \varphi-d \cos \varphi) & 0 & 0 & 0 \\
(r / 2 b) & -(r / 2 b) & 0 & 0 & 0 \\
1 & 0 & 0 & 0 & 0 \\
0 & 1 & 0 & 0 & 0 \\
0 & 0 & 1 & 0 & 0 \\
0 & 0 & 0 & 1 & 0 \\
0 & 0 & 0 & 0 & 1
\end{array}\right]
$$

As well as, $\dot{q}$ can be expressed as follows:

$$
\dot{q}=B(q) v, v=\left[\begin{array}{lllll}
\dot{\theta}_{r} & \dot{\theta}_{l} & \dot{\theta}_{0} & \dot{\theta}_{1} & \dot{\theta}_{2}
\end{array}\right]^{T}
$$

By differentiating of Eq. 10

$$
\ddot{q}=B(q) \dot{v}+\dot{B}(q) v
$$

After performing some algebraic manipulations, the dynamic equation of the mobile manipulator is:

$$
B^{T}(q) \cdot M \cdot(B(q) \dot{v}+\dot{B}(q) v)+B^{T}(q) \cdot(C+G)=B^{T}(q) .
$$

Finally, the dynamic equations in the state space form considered in this paper, are as follow:

$$
\begin{aligned}
\dot{x}=\left[\begin{array}{l}
X_{1} \\
X_{2}
\end{array}\right]=\left[\begin{array}{c}
B v \\
\left(B^{T} M B\right)^{-1}\left(-B^{T} M \dot{B} v-B^{T} C\right)
\end{array}\right] & +\left[\begin{array}{c}
0 \\
\left(B^{T} M B\right)^{-1}
\end{array}\right] F
\end{aligned}
$$




\section{MADL formulation for a given trajectory}

High payload to mass ratio is one of the advantages of robot manipulators. The MADL of a mobile manipulator is defined as the maximum load, which the mobile manipulator can carry in performing the trajectory with acceptable precision for a pre-defined trajectory. The dynamic payload-carrying capacity of a robot manipulator is usually defined as the maximum payload. The maximum MADL, which can be achieved by a manipulator during a given trajectory, is limited by a number of factors. The most important ones are: the dynamic specification of the manipulator, the actuator limitations and the overturning stability. By considering constraints and adopting a logical computing method, the maximum load carrying capacity of a mobile manipulator for a given trajectory can be computed.

\subsection{Formulation of joint actuator torque constraint}

The actuators in a robot are key factors in determining its dynamic performance. Based on the definition of typical torque-speed characteristics of DC motors, the joint actuator torque constant was formulated as follows:

$$
U_{\text {allow }}^{(+)}=c_{1}-c_{2} \dot{q}, \quad U_{\text {allow }}^{(-)}=-c_{1}-c_{2} \dot{q}
$$

where $c_{1}=\tau_{s}, c_{2}=\tau_{s} / \omega_{0}, \tau_{s}$ is the stall torque, $\omega_{0}$ is maximum no load speed of the motor, and $u_{a}^{(+)}$and $u_{a}^{(-)}$are the upper and lower bounds of the allowable torque. The left hand side of both equations above give the upper and the lower allowable torques of any actuators. An experimental mass $\left(m_{e}\right)$, less than maximum estimated load, is then used in order to calculate $\tau_{e}$ for any $i^{t h}$ point along the given trajectory. The allowable torque limits can then be calculated, using the upper and the lower allowable torques of the actuators and $\left(\tau_{e}\right)_{i}$, according to following equations:

$$
\tau_{i}^{(+)}=\left(U_{a}^{(+)}\right)_{i}-\left(\tau_{e}\right)_{i}, \quad \tau_{i}^{(-)}=\left(U_{a}^{(-)}\right)_{i}-\left(\tau_{e}\right)_{i}
$$

The maximum allowable torque of any joint $\left(\tau_{a}\right)_{i}$ can then be calculated as follows:

$$
\left(\tau_{a}\right)_{i}=\max \left\{\tau_{i}^{(+)}, \tau_{i}^{(-)}\right\}
$$

In order to determine the maximum allowable load, by considering the actuator constraint, it is essential to define a load coefficient $\left(c_{a}\right)_{j}$ for any point along the given trajectory as follows:

$$
\left(c_{a}\right)_{j}=\min \left\{\frac{\left(\tau_{a}\right)_{i}}{\max \left(\tau_{e}\right)-\max \left(\tau_{n}\right)}\right\}, \quad i=1,2, \ldots, n
$$


where $\tau_{n}$ represents the no-load torque. Physically, the load coefficient $\left(c_{a}\right)_{j}$ on the $j^{\text {th }}$ joint actuator describes the accessible torque for carrying the maximum load to the torque, which is applied for carrying the initial load.

\subsection{Formulation of stability constraint}

In order to successfully carry out tasks, especially in carrying heavy loads on its trajectory for mobile manipulators, the concepts of the stability degree and the valid stable region become very important to avoid tipping over. Therefore, estimation and evaluation of dynamic stability with an appropriate criterion throughout the motion of such systems is a fundamental necessity. In this investigation, a new tip-over stability measure, named as MomentHeight Stability (MHS) measure will be used, which is given by Moosavian and Alipour [11] for wheeled mobile manipulators. The MHS is defined as an amount of moment, that causes the moving base to rotate around each edge of stability margin, while considering all forces and torques exerted to the base body, due to manipulator motion, gravitational forces, inertial force and external forces/torques.

As shown in Fig. 3, the support boundary with four edges and the base frame $x_{0}, y_{0}, z_{0}$ has been considered for computational aspects. The support boundary polygon (stability margin) is specified as the outermost of the base contact points with the ground. Then, to apply the MHS measure, the resultant moments of all forces and torques exerted to the base about different edges of support polygon are computed. These moments about edges $1,2, \ldots$, and $n$ are named $M_{1}, M_{2}, \ldots, M_{n}$, respectively. The dynamic MHS measure, $\alpha$, is

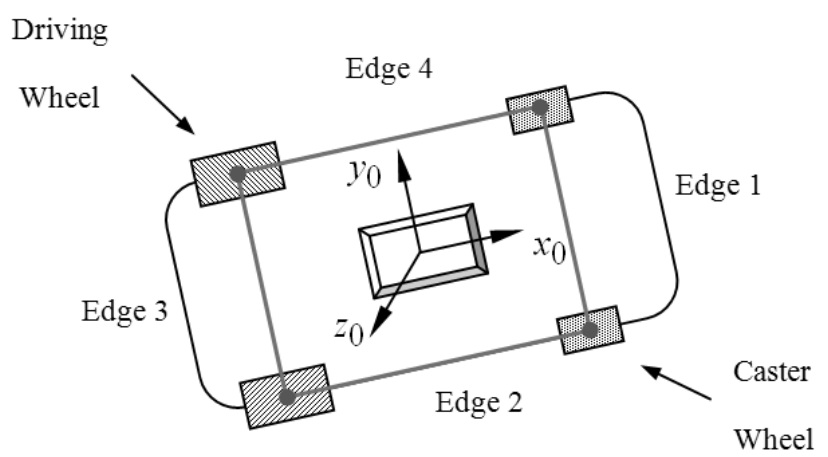

Fig. 3. The support boundary and the base frame 
defined as follows:

$$
\alpha=\min \left(\alpha_{i}\right), \quad i=\{1,2, \ldots, n\}
$$

where $\alpha_{i}$ indicates the dynamic stability margin around the $i^{t h}$ boundary edge and is calculated as:

$$
\alpha_{i}=\left(I_{v i}\right)^{\sigma_{i}} \cdot\left(M_{i} \cdot \widehat{\mathbf{a}}_{i}\right), \quad i=\{1,2, \ldots, n\}
$$

where $\widehat{\mathbf{a}}_{i}$ is the unit vector for each edge of the support polygon and $I_{v i}$ denotes the base moment of inertia around the $i^{t h}$ edge of the rectangular boundary, and:

$$
\sigma_{i}=\left\{\begin{array}{cc}
+1 & \text { if }\left(M_{i} \cdot \widehat{\mathbf{a}}_{i}\right)>0 \\
-1 & \text { otherwise }
\end{array}\right.
$$

when $\alpha$ is positive, the system is stable. On the contrary, negative values of $\alpha$ indicate that a tip-over instability is in progress. Also, critical tip-over stability occurs when $\alpha$ is equal to zero.

For a mobile manipulator, which is able to varying its C. M. height, or carrying a variable payload, the tip-over stability margin must be sensitive to the reduced stability, associated with an increase in the C. M. height. The MHS measure in the above form is not sensitive to changes in system C. M. height. Therefore, the MHS measure $\alpha$ is improved by making it sensitive to changes in system C. M. height, thereby fully capturing the susceptibility to tip-over of the entire system as follows:

$$
\alpha=\left(h_{c . m .}\right)^{\lambda} \cdot \min \left(\alpha_{i}\right), \quad i=\{1,2, \ldots, n\}
$$

where $h_{c . m}$. indicates the system C.M. height, and

$$
\lambda=\left\{\begin{array}{cc}
-1 & \text { if } \min \left(\alpha_{i}\right)>0 \\
+1 & \text { otherwise }
\end{array}\right.
$$

In order to better condition the computational problem and to facilitate interpretation of the measure, the stability measure should be normalized with respect to its nominal value. In this study, the initial situation is considered as nominal configuration. The stability index $\alpha$ can be normalized as follows:

$$
\widehat{\alpha}=\frac{\left(h_{c . m .}\right)^{\lambda}}{\left(h_{c . m .}\right)_{n o m}^{\lambda}} \cdot \frac{\min \left(\alpha_{i}\right)}{\min \left(\alpha_{i}\right)_{n o m}}, \quad i=\{1,2, \ldots, n\}
$$




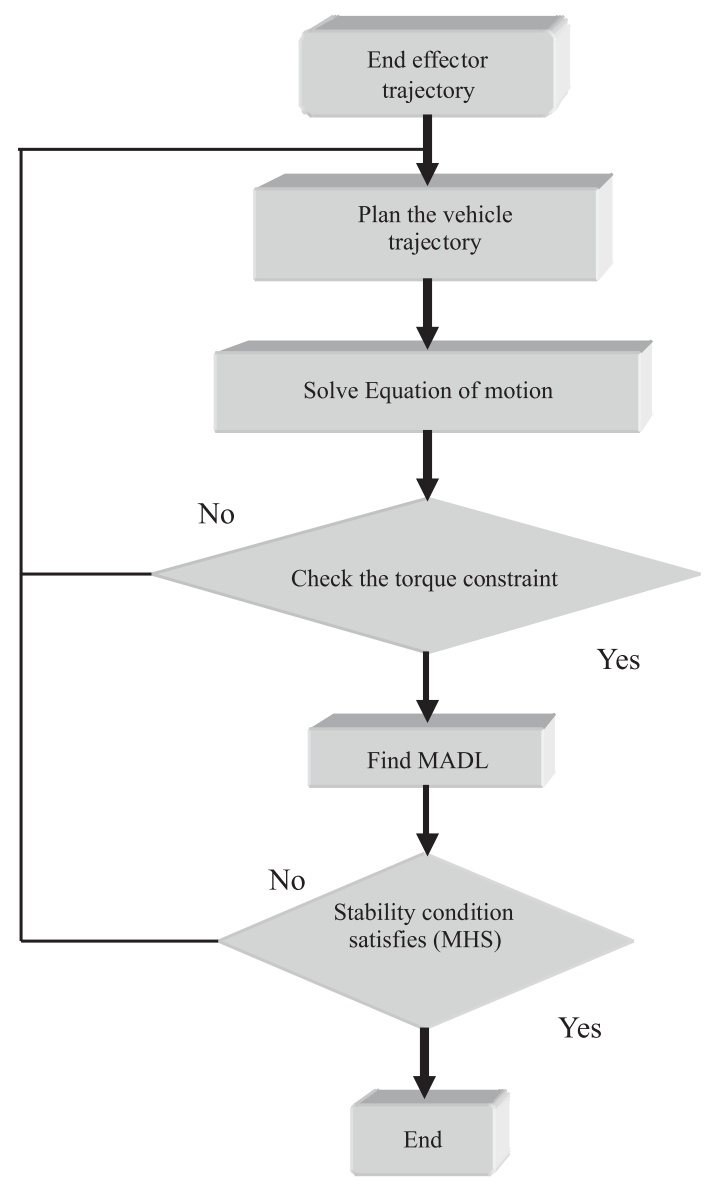

Fig. 4. Flowchart of determining the MADL

where $\widehat{\alpha}$ represents the normalized dynamic stability margin and subscript "nom" refers to the corresponding nominal value. The algorithm of computational procedure for determining maximum allowable load is outlined and also shown in the flowchart in Fig. 4.

\section{Simulation results and discussion}

In this section, two numerical simulations of the mobile manipulator to illustrate the efficiency of the proposed model are performed. One is used to simulate the non-holonomic mobile manipulator, that the end-effector and its load track predefined trajectory, as shown in Fig. 5. In this case, the mobile base of manipulator moves along a clothoid path. In the second one, MADL 
Table 1. Parameters used for simulation

\begin{tabular}{|l|l|l|}
\hline Parameter & Value(Base) & Value(Manipulator) \\
\hline Length of links $(m)$ & $0.8 \times 0.4 \times 0.15$ & $L_{1}=1, L_{2}=0.5$ \\
\hline Mass $(\mathrm{kg})$ & $m_{b}=90$ & $m_{1}=0.8, m_{2}=0.4$ \\
\hline Diameter $(m)$ & - & $D_{o}=0.04, D_{i}=0.035$ \\
\hline Tip mass $(\mathrm{kg})$ & - & $m_{p}=5-15$ \\
\hline
\end{tabular}

is found for the mobile manipulator, in which the mobile base moves along a straight line on sloped terrain. Parameters of the mobile manipulator are given in Table 1.

4.1. MADL of a mobile manipulator moving on a clothoid path

This simulation study is carried out to illustrate the efficiency of the procedure, presented in Fig. 4, for determining the maximum allowable load of a two-links mobile manipulator. The parameters of a simulated two-link mobile manipulator are shown in Table 1.

The desired trajectory of the end-effector and its payload are shown in Fig. 6, which starts from the coordinate $\left(x_{1}=1.3, y_{1}=2.5, z_{1}=0.15 \mathrm{~m}\right)$ to the coordinate $\left(x_{2}=2.9, y_{2}=0.5, z_{2}=0.5 \mathrm{~m}\right)$ with zero initial and final velocity.

As shown in Fig. 7, a clothoid path is planned for the vehicle, which is very useful for smoothing the motion of a mobile robot, moving along a trajectory. An arc of a clothoid has variable curvature, in every point proportional to the arc length, and it provides the smoothest connection between a straight line and a circular curve. The centrifugal force, along a clothoid curve, actually varies in proportion to the time, at a constant rate, from zero value (along the straight line) to the maximum value (along the curve) and back again. The parametric equations of the clothoid are given by the so-called "Fresnel Integrals":

$$
x=\int_{0}^{t} \cos \left(\frac{\pi}{2} t^{2}\right) d t, y=\int_{0}^{t} \sin \left(\frac{\pi}{2} t^{2}\right) d t
$$

Now, the MADL of the mobile manipulator can be determined with respect to pervious section. Both actuators of the robot are taken into account to be the same with $T_{s}=600 \mathrm{~N} . \mathrm{m}$ and $\omega_{n}=10 \mathrm{rad} / \mathrm{s}$. If only the joint actuator torque constraint was imposed, the maximum dynamic load carrying capacity for a given trajectory was found to be $m=17 \mathrm{~kg}$ without considering stability. 


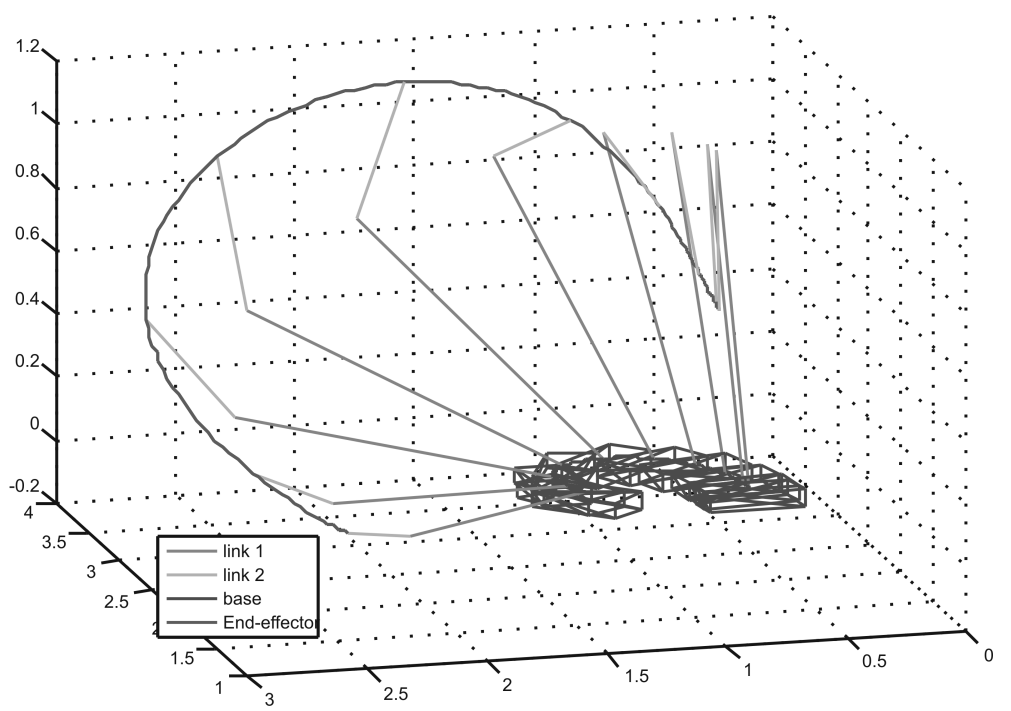

Fig. 5. The response of the system, with various Young's modulus

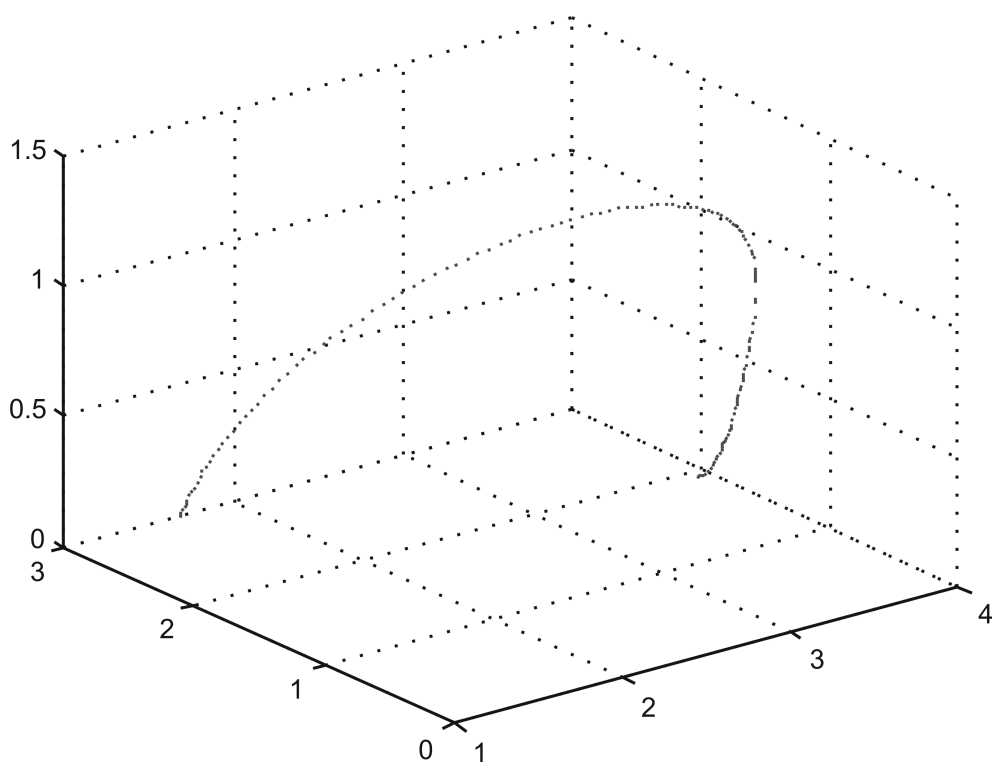

Fig. 6. The desired load path

Figures 8-10 show the corresponding applied torques to the manipulator actuators and wheels within an upper and lower bounds of the available torques. From these results, it can be seen that the loads so determined use the joint 1 


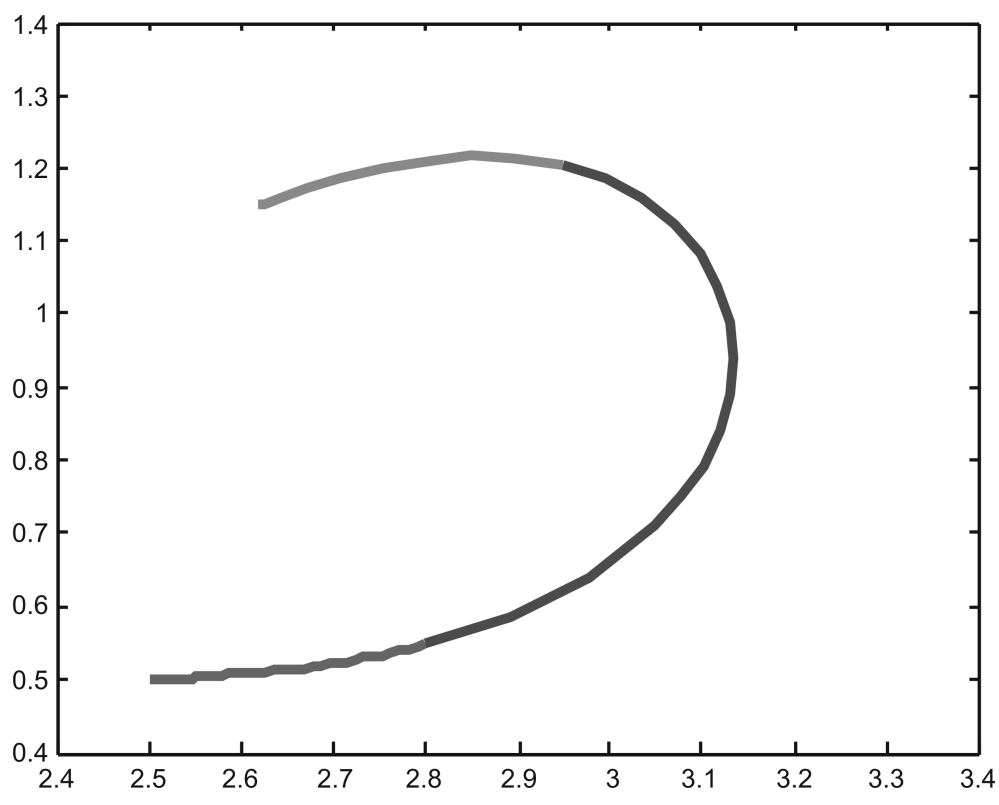

Fig. 7. The planned clothoid path for the vehicle

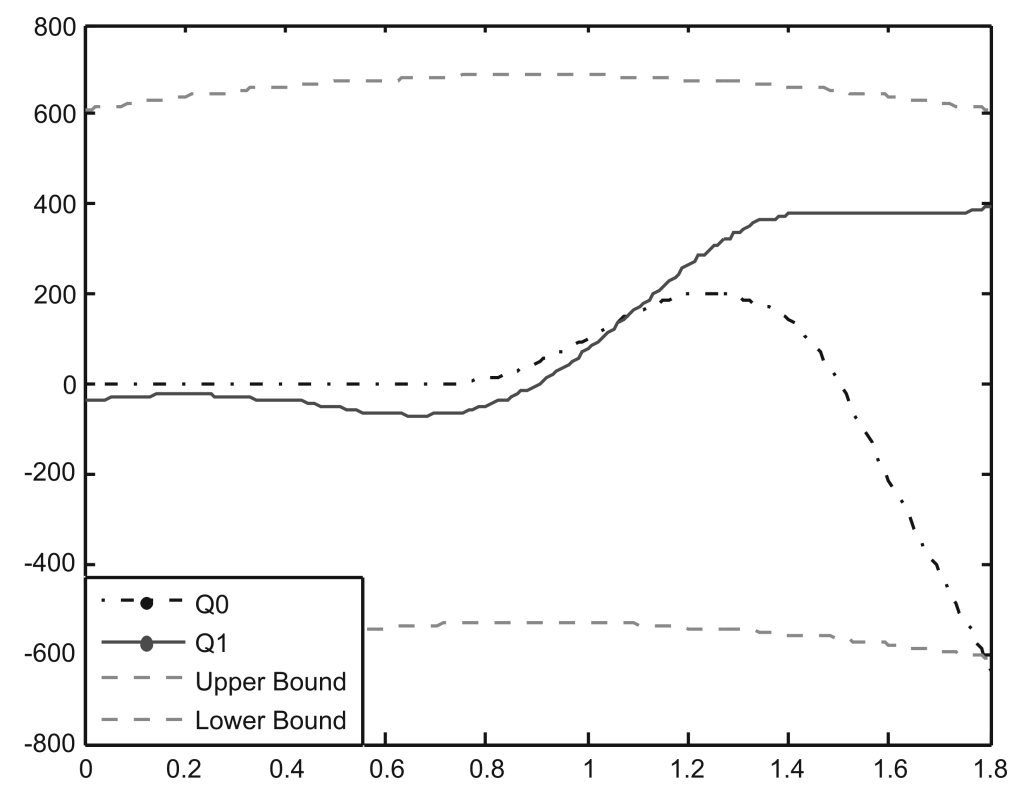

Fig. 8. Actuator torques at the first joint against torque bounds 
Maximum Allowable Dynamic Load of Mobile Manipulators ...

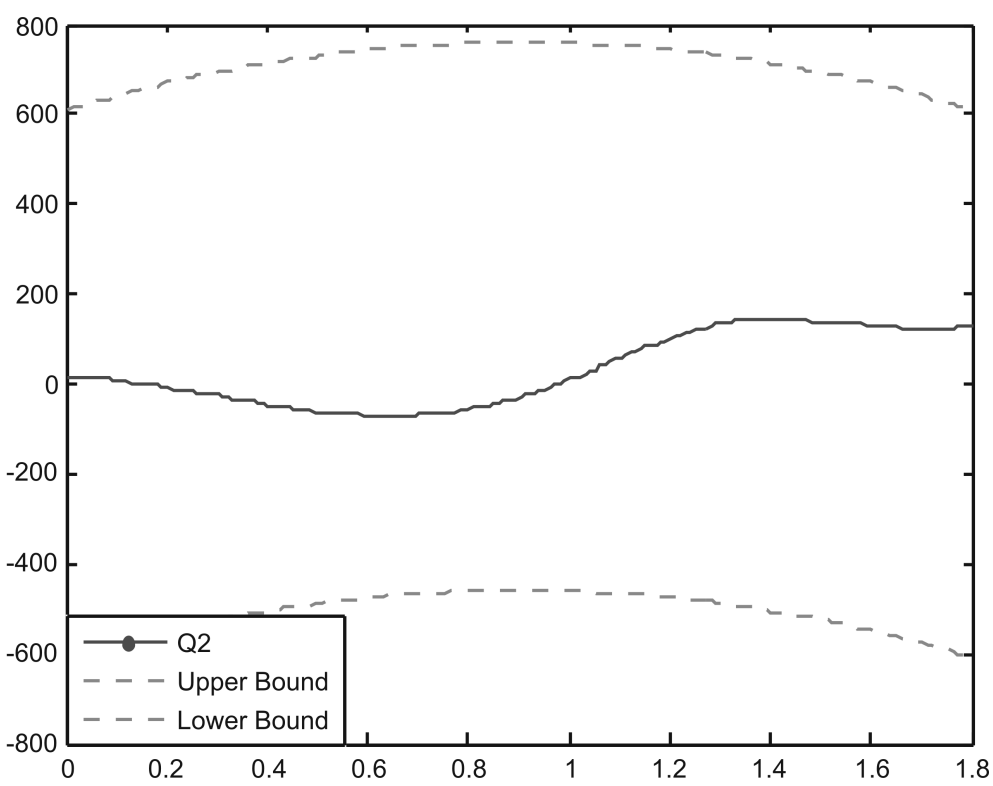

Fig. 9. Actuator torque at the second joint

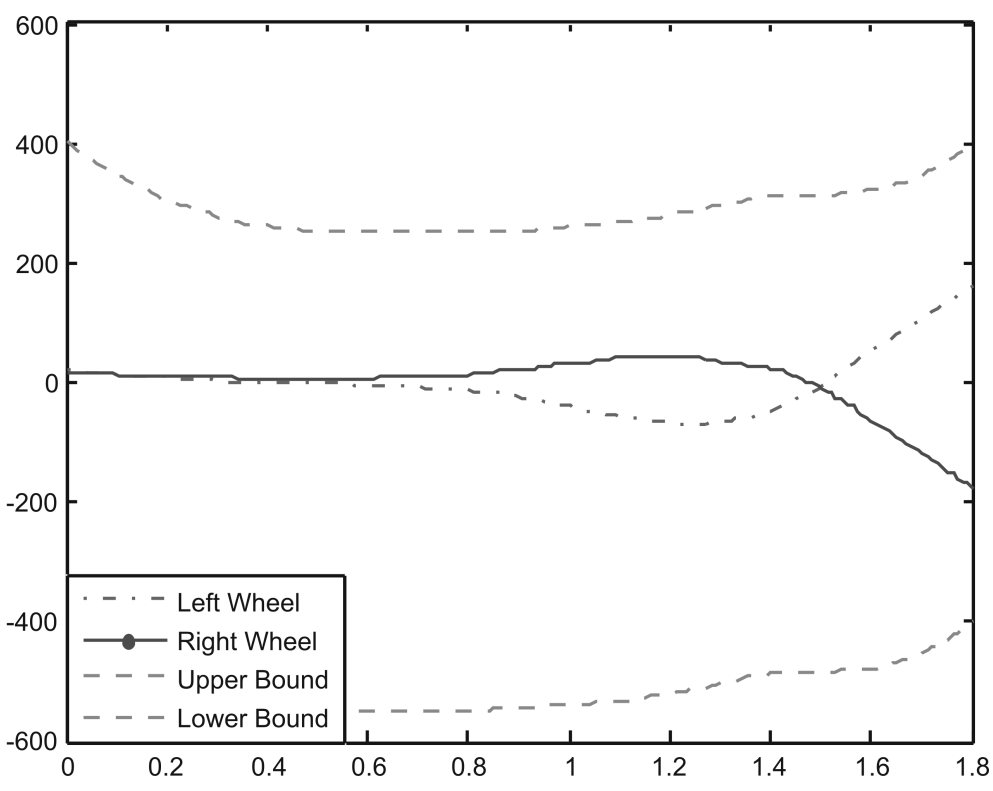

Fig. 10. Actuator torques of the right and left wheels 


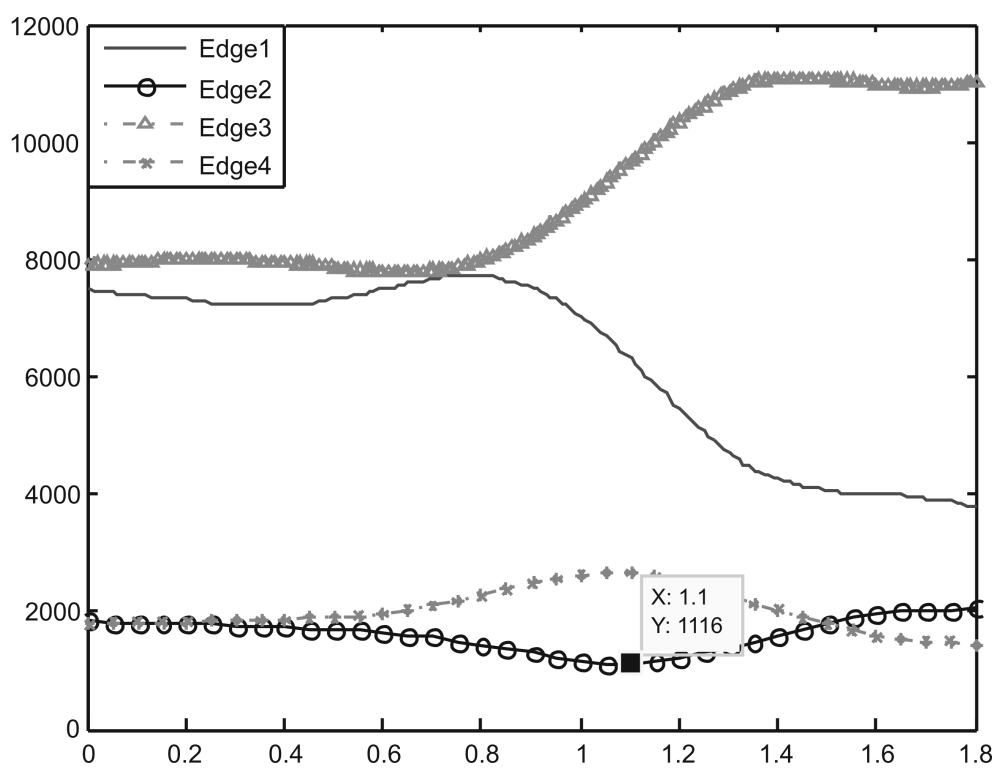

Fig. 11. The MHS criteria associated to the different edges

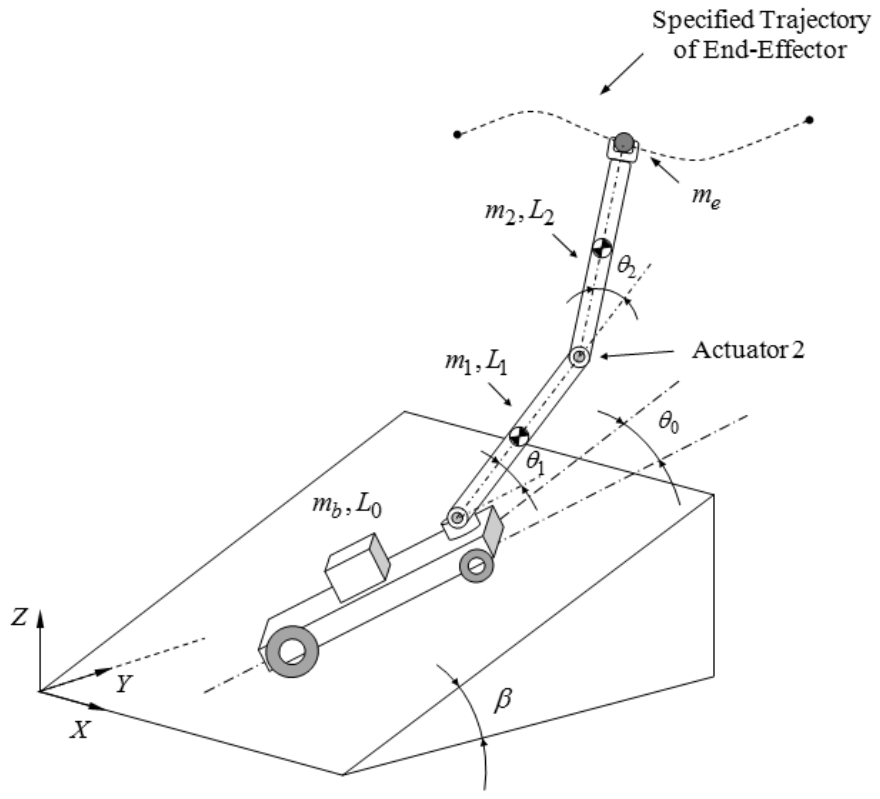

Fig. 12. Schematic of a mobile manipulator moving on a slope terrain 
Maximum Allowable Dynamic Load of Mobile Manipulators ...

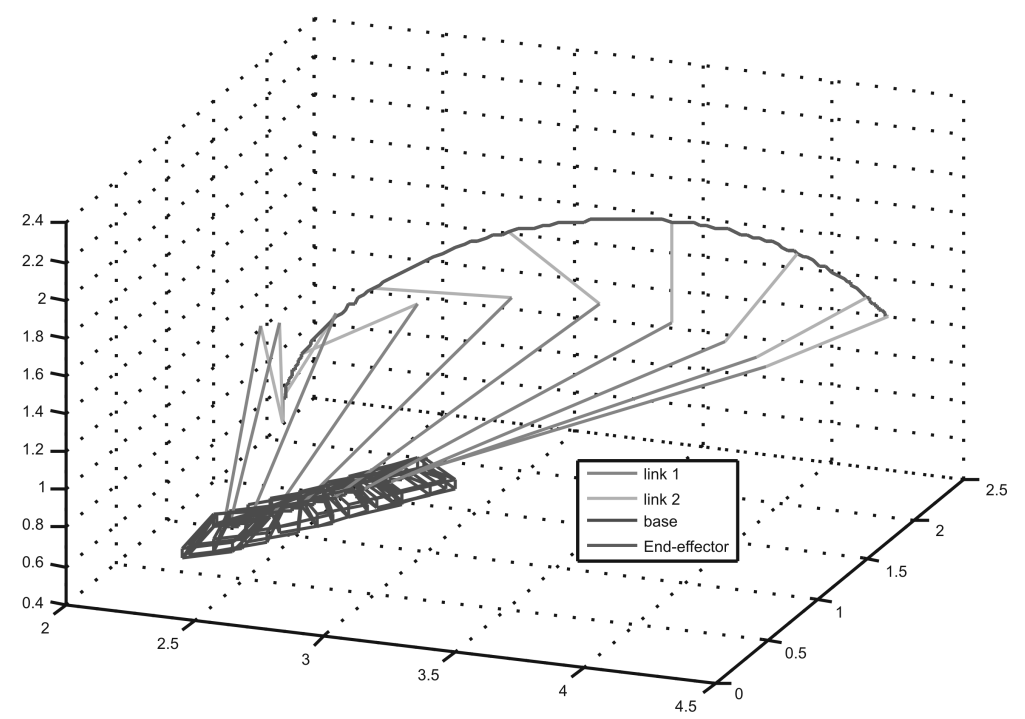

Fig. 13. The desired path of the end-effector

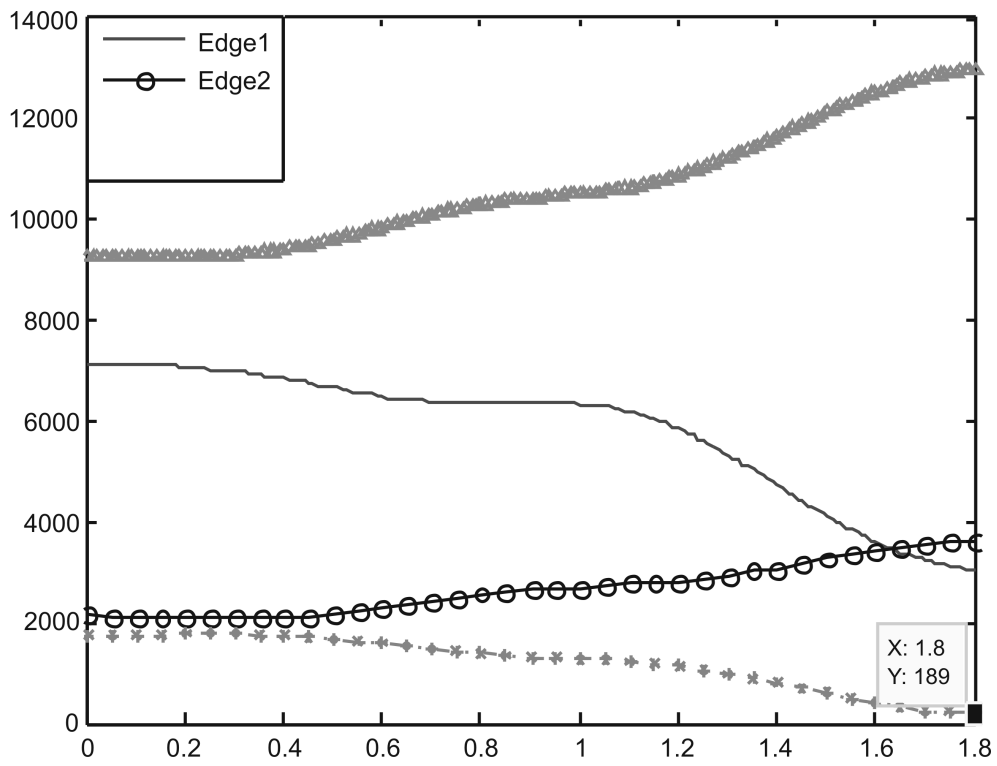

Fig. 14. Variation of the MHS measures related to the different edges of support polygon

to its maximum extent, while the bounds of joint 2 are not reached during the course. 


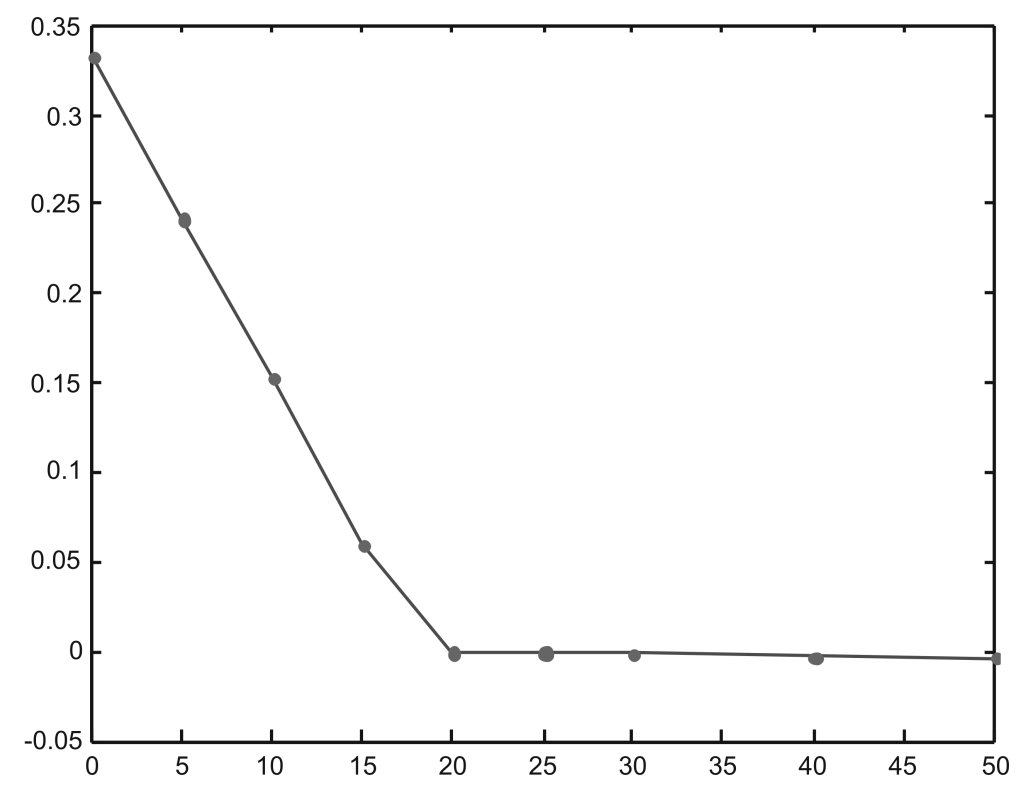

Fig. 15. The variation of normalized stability index for different slope angles

In order to be stable, $\alpha$, must have a positive value for the mobile manipulator. Therefore, the MADL by imposing the stability criterion of the platform must reduce to the safe maximum load to carry, which is $15 \mathrm{~kg}$ for the given trajectory. The magnitude of a positive $\alpha=1116$ describes magnitude of the tip-over stability margin of a stable system. Variation of the MHS measure during such a maneuver, between $t_{i}=0$ and $t_{f}=1.8 \mathrm{~s}$, is shown in Fig. 11. Also, the variation of stability index for different payloads is illustrated in Fig. 12 .

\subsection{MADL of a mobile manipulator moving on a slope}

In most of industrial applications such as factory automation, a mobile manipulator has been used in a flat plane without slope. From a practical point of view, however, it is important to deal with various conditions of the floor surface to increase the reliability and the flexibility of the locomotion. When a mobile robot performs some special tasks, such as carrying a heavy load, moving on a slope or rough terrain, it may become unstable even overturn. In this case study, the computation of the MADL for the mobile manipulator in the motion on a slope (Fig. 12) is presented. The end-effector trajectory is shown in Fig. 13. A linear path is planned for the vehicle, while it is on an inclined plane and the gradient angle of the slope is supposed to be 30 degrees. 
The link parameters and the inertia properties of the mobile manipulator were given in Table 1. Both actuators of the robot are taken into account to be the same as with $T_{s}=600 \mathrm{~N} . \mathrm{m}, \omega_{n 1}=10 \mathrm{rad} / \mathrm{s}$.

If only the joint actuator torque was imposed, the maximum dynamic load carrying capacity for a given trajectory was computed to be $m=15 \mathrm{~kg}$. However, if the stability criterion were added to the above calculations, the stability index, $\alpha=189$, has a positive value. Therefore, the maximum allowable dynamic load of the mobile manipulator with considering both constraints is calculated to be $m=15 \mathrm{~kg}$. Variation of the MHS measure during such a maneuver, between $t=0$ and $t=1.8 \mathrm{~s}$, is shown in Fig. 14. Also, the variation of stability index for different slope angles is illustrated in Fig. 15.

\section{Conclusions}

The main objective of this investigation was determining the maximum allowable dynamic load for mobile manipulators for a given trajectory. The effect of the dynamic interaction between the manipulator and the mobile platform is considered to characterize the motion of a non-holonomic mobile manipulator. The MADL can be obtained by a mobile manipulator during a predefined trajectory are restricted by the number of factors. This was achieved by imposing actuator torque capacity and overturning stability constraints to the problem formulation. Designer should be paying attention to their priority constraints in order to present an ideal path robot. The proposed approach has been implemented on a mobile manipulator and the MADL was obtained. In the two case studies a two-link mobile manipulator was considered for carrying a load on the clothoid path and the slope terrain. The Numerical results of the two case studies are shown by changing the vehicle motion for a pre-defined end effector trajectory or initial configuration of the mobile manipulator, the stability condition is assured for calculating the MADL. In addition, the formulation is more stable and efficient than most alternatives and has the added advantage that it can be calculated stability constraint. Moreover, the MHS stability criterion is sensitive to such variation of $\mathrm{C}$. M. position and provides the specific indication of instability occurrence, especially in manipulating heavy objects.

\section{REFERENCES}

[1] Arakawa, T., T. FukudA. Natural Motion Generation of a Biped Locomotion Robot using the Hierarchical Trajectory Generation Method Consisting of GA, 
EP Layers, Proc. of the IEEE Conf. on Robotics and Automation, 1 (1997), 211-216.

[2] Chen, M. W., A. M. S. Zalzala. Dynamic Modelling and Genetic-base Trajectory Generation for Non-holonomic Mobile Manipulators. Control Engineering Practice, 5 (1997), 39-48.

[3] Ghasempoor, A., N. Sepenri. A Measure of Stability for Mobile Manipulators with Application to Heavy-duty Hydraulic Machines. Transactions of ASME, Journal of Dynamic System, Measurement and Control, 120 (1998), 360-370.

[4] Goswami, A. Postural Stability of Biped Robots and the Foot Rotation Indicator (FRI) Point. International Journal of Robotics Research, 18 (1999), 523-533.

[5] Huang, Q., S. Sugano. Motion Planning of Stabilization and Cooperation of a Mobile Manipulator, Proc. IEEE/IROS, 2 (1996), 568-575.

[6] Kim, J., W. K. Chung, Y. Youm, B. H. Lee. Real-time ZMP Compensation Method using Null Motion for Mobile Manipulators, Proc. of the IEEE Conf. on Robotics and Automation, 2 (2002), 1967-1972.

[7] Korayem, M. H., H. Ghariblu. Maximum Allowable Load on Wheeled Mobile Manipulators imposing Redundancy Constraints. J. of Robotic and Autonomous Systems, 44 (2003), 151-159.

[8] Korayem, M. H., A. Heidari. Maximum Allowable Dynamic Load of Flexible Mobile Manipulators using Finite Element Approach. Int. J Adv Manufacture and Technology, 36 (2007), 1010-1021.

[9] LI, Y. Dynamic Stability Analysis and Control for the Mobile Manipulator, Proc. of the IEEE Conf. on Electrical \& Computer Engineering, 1 (2002), 554-559.

[10] Messuri, D., C. A. Klein. Automatic Body Regulation for Maintaining Stability of a Legged Vehicle during Rough-terrain Locomotion. IEEE, J. Robotics and Automation, 1 (1985), 132-141.

[11] Moosavian, S. A. A., K. Alipour. On the Dynamic Tip-over Stability of Wheeled Mobile Manipulators. International Journal Robotics and Automation, 22 (2007), 1-7.

[12] Papadopoulos, E. G., D. A. Rey. The Force-angle Measure of Tip-over Stability Margin for Mobile Manipulators. Vehicle System Dynamics, 33 (1998), 29-48.

[13] Seraji, H. An Unified Approach to Motion Control of Mobile Manipulators. The International Journal of Robotics Research, 17 (1998), 107-118.

[14] Wang, L. T., B. Ravani. Dynamic Load Carrying Capacity of Mechanical Manipulators - Part I: Problem Formulation. Transactions of ASME, Journal of Dynamic System, Measurement and Control, 110 (1988), 46-52.

[15] Yамамото, Y., X. Yun. Coordinating Locomotion and Manipulation of a Mobile Manipulator. IEEE Transactions on Robotics and Automation, 39 (1994), 1326-1332. 